\section{Parâmetros ambientais e granulométricos nas praias de Cabuçu e Bom Jesus dos Pobres no Recôncavo da Bahia}

\author{
Environmental and particle size parameters on Cabuçu and Bom \\ Jesus dos Pobres beaches, in Recôncavo Bahiano, Brazil
}

Irana Paim Silva ${ }^{[a]}$, Elinsmar Vitória Adorno ${ }^{[\mathrm{b}]}$

\section{Resumo}

0 objetivo deste estudo é a avaliação de parâmetros ambientais como temperatura, oxigênio, salinidade, pH e a granulometria, que podem influenciar a composição e distribuição dos organismos marinhos. 0 trabalho foi realizado nas praias de Cabuçu (CAB) e Bom Jesus dos Pobres (BJP), situadas no Recôncavo da Bahia. Os valores da temperatura em $\mathrm{CAB}$ variaram de $27,27^{\circ} \mathrm{C}$ (jun/2009) a $34,65^{\circ} \mathrm{C}$ (dez/2008) e em BJP de $26,44^{\circ} \mathrm{C}$ (jun/2009) a $37,27^{\circ} \mathrm{C}$ (dez/2008); essas variações foram associadas ao clima da região. Os valores mínimo e máximo dos níveis de oxigênio dissolvido em $C A B$ foram iguais a 14,5\% (jun/2009) e 55,9\% (dez/2008); em BJP, esse mesmo parâmetro variou entre 21,8\% (jun/2008) e $42 \%$ (abr/2009). Em ambas as localidades, essas variações podem estar associadas à energia das praias. A salinidade em CAB esteve entre 23,54\% (abr/2009) a 37,04\% (out/2008) e em BJP de 28,45\% (fev/2009) a 36,04\% (abr/2009), estando a média dentro dos padrões para águas salinas. Para o pH, CAB apresentou 8,38 $\mathrm{H}^{+}$(jun/2009) e 8,12(dez/2009). Já BJP, $8,09 \mathrm{H}^{+}$(ago/2009) e 8,44 $\mathrm{H}^{+}$(dez/2008). As médias encontradas estiveram abaixo dos valores referidos pela Resolução CONAMA, mas dentro da média estabelecida para águas salinas. Assim, os valores encontrados para os parâmetros ambientais analisados foram considerados normais. As medidas granulométricas da areia da praia determinaram que a fração predominante foi areia média, sendo que $C A B$ apresentou pouca variação no diâmetro das partículas e um maior grau de selecionamento. Por outro lado, em BJP houve uma maior variação de diâmetro das partículas em relação à CAB.

Palavras-chave: Composição e distribuição dos organismos marinhos. Parâmetros ambientais de praias. Recôncavo. Sedimento.

\begin{abstract}
The aim of this study was to assess environmental parameters as temperature, oxygen, salinity, pH and particle size which can influence the composition and distribution of marine organisms. The samplings were made on the beaches of Cabuçu $(C A B)$ and Bom Jesus dos Pobres (BJP) both located in a geographical region known as Recôncavo Bahiano, State of Bahia, Brazil. The temperature at $C A B$ ranged from $27.27^{\circ} \mathrm{C}$ (June/2009) to $34.65^{\circ} \mathrm{C}$ (December/2008). Similarly, the temperature at BJP varied from $26.44^{\circ} \mathrm{C}$ (June/2009) to $37.27^{\circ} \mathrm{C}$ (December/2008). In both localities the changes were associated to the climate of the region. Variation ranges of dissolved oxygen at CAB were from $14.5 \%$ (December/2008) to $55.9 \%$ in (June/2009) and at BJP from 21.8\% (June/2008) to 42\% in (April/2009) and they may be associated to energy input from rivers and beaches. Salinity in CAB was between $23.54 \%$ (April/2009) and $37.04 \%$ (October/2008) and in BJP from 28.45\% (February/2009) to 36.04\% (April/2009), and the average was within the standards for saline waters. Regarding $\mathrm{pH}, \mathrm{CAB}$ showed $8.38 \mathrm{H}^{+}$(June/2009) and 8.12 (December/2009) and BJP $8.09 \mathrm{H}^{+}$(August/2009) and $8.44 \mathrm{H}^{+}$(December/2008), values found were below the averages of the values specified by CONAMA Resolution, but within the range established for saline water. The environmental parameter values were considered normal. The sand particle size analysis indicated that the predominant fraction in the sieves was medium sand. CAB showed little variation in particle diameter and a higher degree of sorting, while BJP showed a greater variation in particle diameter.
\end{abstract}

Keywords: Composition and distribution of marine organisms. Environmental parameters. Recôncavo. Sediment.
${ }^{[a]}$ Bióloga pela Universidade Federal do Recôncavo da Bahia (UFRB), Cruz das Almas, BA - Brasil, e-mail: anaripaim@hotmail.com

${ }^{[b]}$ Biólogo pela Universidade Federal da Bahia, Mestre, professor assistente Centro de Ciências Agrárias, Ambientais e Biológicas, Universidade Federal do Recôncavo da Bahia (UFRB), Cruz das Almas, BA - Brasil, Câmpus Universitário, e-mail: adornos@ufrb.edu.br

Recebido: $16 / 02 / 2010$ Received: 02/16/2010

Aprovado: 19/05/2010 Approved: 05/19/2010 


\section{Introdução}

As praias constituem sistemas dinâmicos, em que elementos básicos como ventos, água e areia interagem, resultando em processos hidrodinâmicos e deposicionais complexos (1). A morfologia dos perfis praiais em uma determinada região, é função do nível energético das ondas, uma vez que essa energia é liberada nas zonas costeiras. Neste sentido, quanto ao grau de exposição, as praias podem ser identificadas desde muito expostas a muito protegidas, sendo a variabilidade física resultante da combinação de parâmetros básicos como característica das ondas e granulometria do sedimento (2).

Os fatores ambientais como temperatura, salinidade e ação de ondas atuam de maneira uniforme sobre o ambiente. Destes, o fator mais importante é a ação das ondas, que, por sua vez, influi no tamanho do grão de areia, causando variação na retenção de água e a motilidade do substrato. Tais fatores vão influenciar a facilidade de escavação pelos animais, o espaço disponível para habitação, o acúmulo de matéria orgânica e também o tamanho dos organismos intersticiais. Em geral, sedimentos mais finos proporcionam ambiente mais favorável aos seres vivos, principalmente porque possuem maior umidade e maior acúmulo de matéria orgânica enquanto os mais grossos, ao contrário, limitam a existência da infauna. Assim, praias planas, constituídas de areia muito finas e onde quebram ondas pequenas, possuem uma biomassa animal muito superior à das praias inclinadas, cujas areias sejam grossas e fortes o embate de ondas (3).

Este estudo tem como objetivo caracterizar e estimar comparativamente os parâmetros físico-químicos e granulométricos das praias de Bom Jesus dos Pobres e Cabuçu, BA.

\section{Material e métodos}

\section{Área de estudo}

O Recôncavo Baiano está localizado no entorno da Baía de Todos os Santos (Figura 1), possuindo características de clima tropical. A região é muito rica em petróleo e na agricultura da cana-de-açúcar graças ao seu solo e características climáticas. É composta de 33 municípios e tem como limite norte a cidade de Salvador.

A praia de Cabuçu $\left(12^{\circ} 47^{\prime} \mathrm{S}-38^{\circ} 46^{\prime} \mathrm{W}\right)$ é um destino muito requisitado no verão por banhistas.
Apresenta-se com um mesolitoral de aproximadamente $100 \mathrm{~m}$, uma praia com pouca inclinação e substrato predominantemente lamoso. Já a praia de Bom Jesus dos Pobres (1248’56,3"S e 3846'36,2" W), é plana e arenosa com um mesolitoral aproximadamente de $300 \mathrm{~m}$, falésias, áreas de manguezais e de Floresta Atlântica com rios e cascatas. Ambas as praias estão localizadas no Município de Saubara e são utilizadas no extrativismo de moluscos.

As praias de Cabuçu(CAB) e Bom Jesus dos Pobres (BJP) possuem uma distância entre si em média de 3 $\mathrm{km}$. Apesar da pouca distância que as separa, apresentam características paisagísticas distintas e uma fauna bentônica bem adaptada.

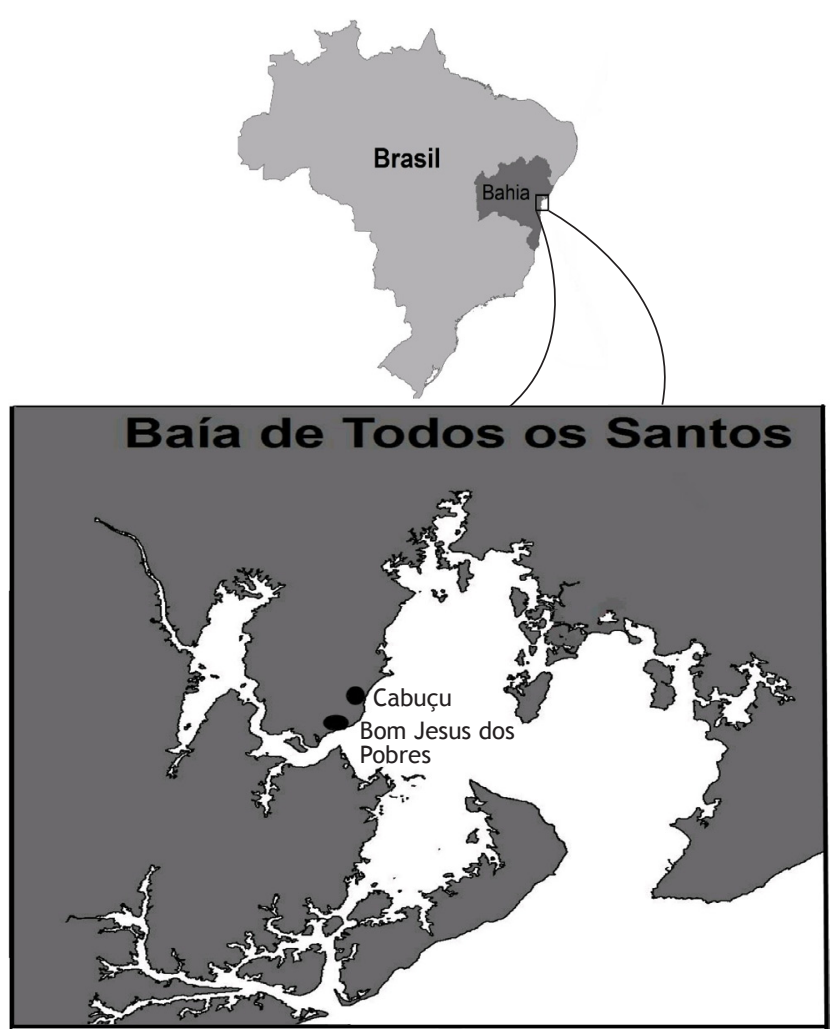

Figura 1 - Localização geográfica da área de estudo (1) Cabuçu, e a área de estudo (2) Bom Jesus dos Pobres - Bahia/Brasil

Fonte: Material cedido pelo Lameb - Universidade Federal da Bahia-UFBA.

\section{Coleta e análise do material}

Foram realizadas seis campanhas bimestrais ao longo de um ciclo anual, entre outubro de 2008 a setembro de 2009 nas praias de Cabuçu e Bom Jesus dos Pobres. A análise Físico-Química foi realizada na 
maré baixa, com marés preferencialmente de sizígia. Os parâmetros foram aferidos com a utilização da Sonda Multiparâmetros (Hanna HI 9828) (4). No mês de abril de 2010, foram coletados $1.000 \mathrm{~g}$ de areia de ambas as praias no segmento do mesolitoral. 0 material foi devidamente ensacado, etiquetado e levado ao Núcleo de Engenharia de Água e Solo - NEAS na Universidade Federal do Recôncavo da Bahia.

\section{Procedimentos de laboratório}

Os dados coletados por meio da Sonda Multiparâmetros (Hanna HI 9828) foram devidamente cadastrados, organizados e plotados em gráficos para posterior avaliação e comparação entre as duas praias.

As análises granulométricas foram realizadas no Laboratório de Solos (NEAS) da Universidade Federal do Recôncavo da Bahia. Para a identificação das frações granulométricas foram utilizados $200 \mathrm{~g}$ do material coletado. Os sedimentos passaram pelos procedimentos iniciais de lavagem para retirada dos sais solúveis, secagem, sendo realizada em estufa à temperatura de $40^{\circ} \mathrm{C}$ por $4 \mathrm{~h}(5)$. Em seguida, visando a distribuir as partículas do sedimento segundo seu tamanho, foi utilizado um conjunto de 10 peneiras padrão da Série Tyler de acordo com as normas da ABNT. A amostra na peneira do topo é agitada durante $15 \mathrm{~min}$. Posteriormente, pesou-se, com auxílio de uma balança analítica, a massa do sedimento retida em cada peneira (6).

\section{Resultados e discussão}

\section{Parâmetros abióticos}

A caracterização dos parâmetros Físico-Químicos no ambiente marinho, revela as condições ambientais que as populações locais enfrentam, podendo as variações afetarem os organismos em seus processos reprodutivos ou no seu desenvolvimento, levando-os a adaptações fisiológicas únicas (7).

\section{Temperatura}

A praia de $\mathrm{CAB}$ apresentou uma variação de temperatura menor se comparado a BJP $27,27^{\circ} \mathrm{C}$ (jun/2009) a $34,65{ }^{\circ} \mathrm{C}(\mathrm{dez} / 2008)$. Já a praia de BJP, apresentou uma grande variação de temperatura $26,44{ }^{\circ} \mathrm{C}$ (jun/2009) a $37,27^{\circ} \mathrm{C}$ (dez/2008). Essa variação pode estar vinculada aos meses de inverno e verão respectivamente, associada à sazonalidade (Gráfico 1), a temperatura é provavelmente o parâmetro mais importante a ser medido, pois, juntamente com a salinidade, determinará a densidade e os padrões de circulação oceânicos (3), controlando a distribuição e as atividades de animais e plantas, agindo como um fator limitante à reprodução, ao crescimento e à distribuição de organismos (8).

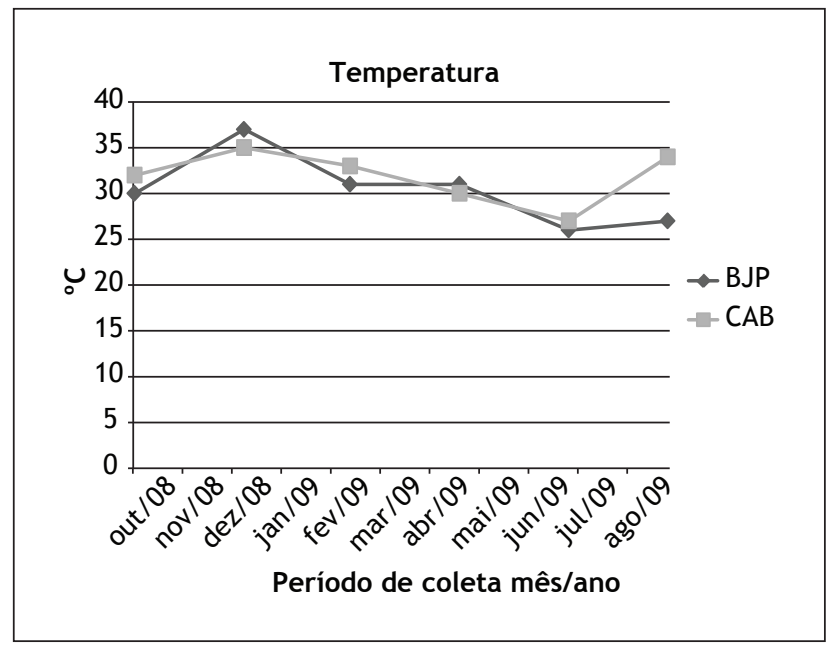

Gráfico 1 - Gráfico de Temperatura das praias de Bom Jesus dos Pobres e Cabuçu - Bahia/Brasil

Fonte: Dados da pesquisa.

Os valores encontrados para as praias de BJP e CAB são considerados normais para o tipo de região, apresentando um clima tropical predominantemente quente. A Resolução Conama para águas salinas não oferece um padrão ou uma média de temperatura ideal. Nos organismos, a elevação da temperatura das águas oceânicas provoca a morte como é o caso de algumas espécies de corais da costa brasileira que sofrem o fenômeno conhecido como branqueamento.

\section{Oxigênio}

A disponibilidade do oxigênio foi inversamente proporcional à temperatura. Elevações de temperatura reduzem o nível de oxigênio, assim como a sua redução aumenta a quantidade de oxigênio dissolvido. Os valores encontrados para o oxigênio em BJP foram 
de: 15\% (ago/2009) valor mínimo; 42\% (abr/2009) valor máximo. Já em CAB no mesmo período: 14,5\% (jun/2009) valor mínimo; 55,9\% (dez/2008) valor máximo (Gráfico 2). No entanto, não foi identificada uma relação entre a temperatura e o oxigênio.

A quantidade de oxigênio dissolvido na água é um dos principais indicadores da saúde dos ecossistemas aquáticos. Se a sua concentração baixar demasiadamente, os organismos com mobilidade irão abandonar o local (sempre que possível), enquanto que os animais sésseis entrarão em stress e eventualmente morrerão se as condições não se alterarem, ou seja, o aumento da concentração de oxigênio (9).

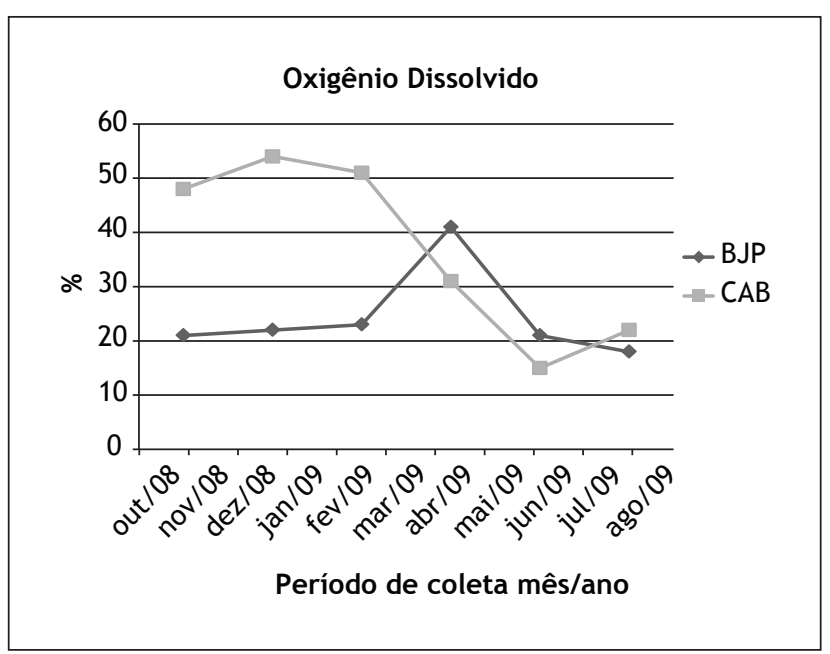

Gráfico 2 - Gráfico de oxigênio dissolvido das praias de Bom Jesus dos Pobres e Cabuçu - Bahia/Brasil Fonte: Dados da pesquisa.

\section{Salinidade}

A salinidade para $\mathrm{CAB}$ apresentou os seguintes valores: 23,54\% (abr/2009) valor mínimo; 37,04\% (out/2008) valor máximo. Já a praia de BJP apresentou salinidade de 28,45\% (fev/2009) valor mínimo; 36,04\% (abr/2009) valor máximo (Gráfico 3). De acordo com Schmiegelow (3), é considerada normal, já que a média de salinidade das águas costeiras é de $35 \%$ e a média encontrada para BJP foi de $34 \%$ e CAB foi de $32,8 \%$, podendo ser explicado em virtude da Baía de Todos os Santos ser uma baía aberta e com características de águas oceânicas. Define-se a salinidade da água do mar pela concentração de todos os sais dissolvidos por litro de água (10). Variações nos gradientes de salinidade e de temperatura, podem definir a composição e a estrutura de assembleias zooplanctônicas, assim como a abundância e a biomassa (11). Farias (8) explica que as pequenas variações locais na salinidade superficial do mar ocorrem devido às chuvas, evaporação e atividade biológica consumidora de sais. Com a redução da salinidade, verifica-se a morte de organismos bentônicos por afetar o equilíbrio homeostático.

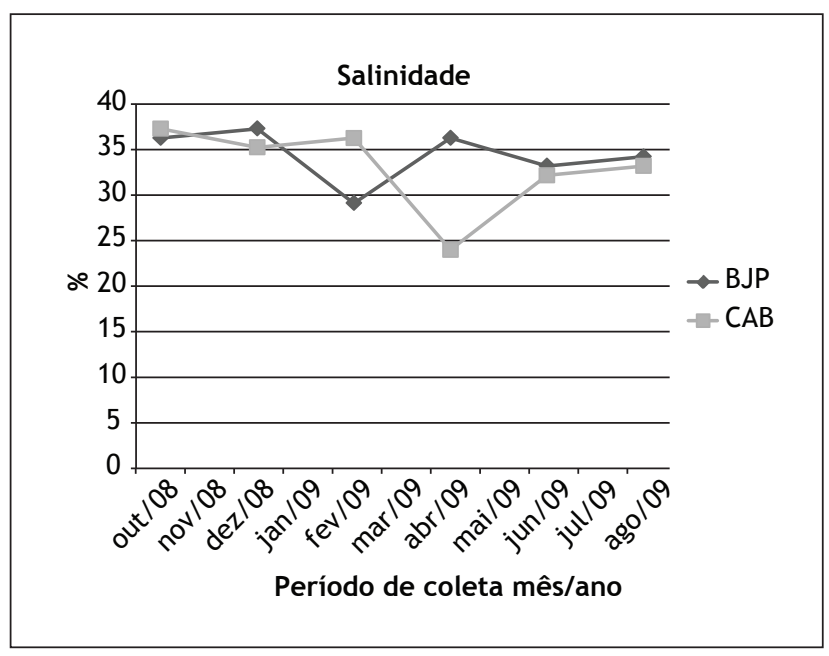

Gráfico 3 - Gráfico de salinidade das praias de Bom Jesus dos Pobres e Cabuçu - Bahia/Brasil

Fonte: Dados da pesquisa.

$\mathrm{pH}$

A praia de Cabuçu (CAB) apresentou uma média de $\mathrm{pH}$ de 8,3 , tendo variações entre máxima de 8,38 no mês de jun/2009 e mínima de 8,12 no mês de dez/2009. Já a praia de Bom Jesus dos Pobres (BJP), apresentou uma média de $\mathrm{pH}$ de 8,32 , com valor máximo no mês de dez/2008 de 8,44 e valor mínimo de 8,09 no mês de ago/2009 (Gráfico 4). Os oceanos são um enorme tanque para o dióxido de carbono lançado na atmosfera. Ao serem emitidos, os gases de efeito estufa são absorvidos em parte pela água marinha, os íons de hidrogênio acabam deixando o oceano mais ácido. Uma mudança de pH de 0,1 pode deixar as águas oceânicas 10 vezes mais ácidas, o que tira de muitos organismos marinhos a habilidade de produzirem as conchas que os protegem, além de causar alterações na cadeia alimentar (12). 


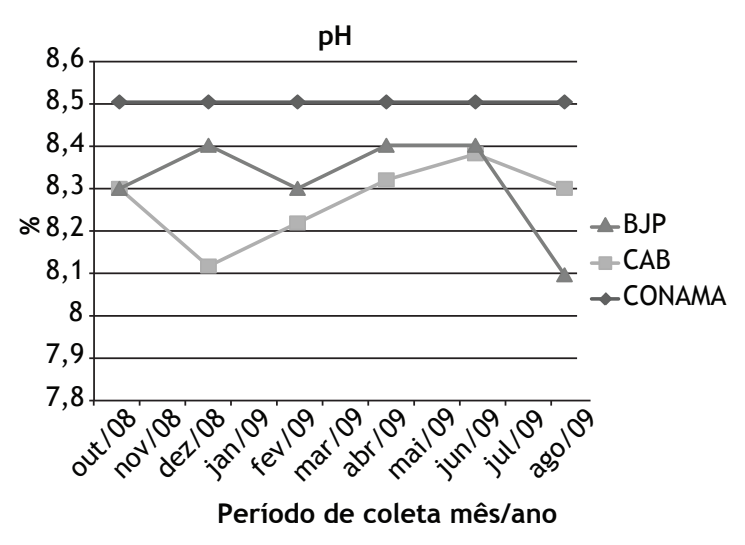

Gráfico 4 - Gráfico de $\mathrm{pH}$ das praias de Bom Jesus dos Pobres e Cabuçu - Bahia/Brasil

Fonte: Dados da pesquisa.

Ambas as praias apresentaram o seu potencial hidrogeniônico baixo, se comparado com a Resolução Conama para águas salinas, que é de 8,5. Mas, de acordo com o que Farias (8) apresentou, a água do mar tem um $\mathrm{pH}$ entre 8,0 e 8,3, significando uma alcalinidade do oceano. 0 aumento na acidez da água do mar pode consumir o exterior da superfície das conchas dos moluscos e outros organismos calcificados, o que poderia levar à morte algumas espécies ou torná-las mais vulneráveis a novos predadores.

\section{Distribuição Granulométrica}

Para as medidas de granulometria avaliadas, a fração predominante nas peneiras foi areia média. Os resultados obtidos estão expostos na Tabela 1.

Tabela 1 - Análise granulométrica da areia da praia de Cabuçu - Bahia/Brasil, distribuída de acordo com o número da peneira e a abertura da malha, sendo demonstrados a massa retida e o diâmetro médio das partículas

\begin{tabular}{llll}
\hline $\begin{array}{l}\text { Número da } \\
\text { peneira }\end{array}$ & $\begin{array}{l}\text { Abertura } \\
(\mathrm{mm})\end{array}$ & $\begin{array}{l}\text { Massa retida } \\
(\mathrm{g})\end{array}$ & $\begin{array}{l}\text { Diâmetro } \\
\text { médio }(\mathrm{g})\end{array}$ \\
\hline 9 & 2,00 & 0,249 & 0,00 \\
16 & 1,00 & 1,247 & 1,5 \\
32 & 0,500 & 167,237 & 0,750 \\
65 & 0,212 & 23,433 & 0,356 \\
80 & 0,180 & 1,486 & 0,196 \\
150 & 0,106 & 4,343 & 0,143 \\
\hline
\end{tabular}

Tabela 1 - Análise granulométrica da areia da praia de Cabuçu - Bahia/Brasil, distribuída de acordo com o número da peneira e a abertura da malha, sendo demonstrados a massa retida e o diâmetro médio das partículas

\begin{tabular}{llll}
\hline $\begin{array}{l}\text { Número da } \\
\text { peneira }\end{array}$ & $\begin{array}{l}\text { Abertura } \\
(\mathbf{m m})\end{array}$ & $\begin{array}{l}\text { Massa retida } \\
(\mathbf{g})\end{array}$ & $\begin{array}{l}\text { Diâmetro } \\
\text { médio }(\mathbf{g})\end{array}$ \\
\hline 270 & 0,270 & 0,236 & 0,188 \\
PAN & 0,00 & 0,00 & 0,135 \\
\hline Total & & 198,233 & \\
\hline
\end{tabular}

Fonte: Dados da pesquisa.

A granulometria evidencia-se como um dos mais importantes fatores na distribuição da meio/fauna, determinando diretamente o tipo e a quantidade dos organismos que a compõem, e indiretamente a qualidade de alimento disponível (13).

Os sedimentos encontrados na praia de Cabuçu apresentaram pouca variação de diâmetro. Percebese, claramente, uma grande concentração de massa na peneira de 0,500 $\mathrm{mm}$ e há um maior grau de seleção em relação a Bom Jesus dos Pobres, estando os grãos distribuídos entre os diâmetros de 0,750 mm e 0,356 (Tabela 1). De acordo com a escala da ABNT para a areia, é caracterizada como uma areia grossa à média.

0 sedimento encontrado na Praia de Bom Jesus dos Pobres apresentou maior variação nos diâmetros dos grãos. Percebe-se, claramente, uma grande concentração de massa na peneira de $0,212 \mathrm{~mm}$, e há um menor grau de selecionamento em relação a Cabuçu, estando os grão distribuídos entre os diâmetros de $0,750 \mathrm{~mm}$ e $0,143 \mathrm{~mm}$ (Tabela 2). De acordo com Tominaga (6), pela classificação Gems Water (1978), estando os sedimentos distribuídos entre os diâmetros $0,425 \mathrm{~mm}$ a $0,163 \mathrm{~mm}$, é considerado de areia média a fina. E, segundo a ABNT, é considerada areia média.

Tabela 2 - Análise Granulométrica da areia da praia de Bom Jesus dos Pobres - Bahia/Brasil, distribuída de acordo com o número da peneira e a abertura da malha, sendo demonstrados a massa retida e o diâmetro médio das partículas

(continua)

\begin{tabular}{llll}
\hline $\begin{array}{l}\text { Número da } \\
\text { peneira }\end{array}$ & $\begin{array}{l}\text { Abertura } \\
(\mathrm{mm})\end{array}$ & $\begin{array}{l}\text { Massa retida } \\
(\mathrm{g})\end{array}$ & $\begin{array}{l}\text { Diâmetro } \\
\text { médio }(\mathrm{mm})\end{array}$ \\
\hline 9 & 2,00 & 0,113 & 0,00 \\
16 & 1,00 & 0,198 & 1,50 \\
32 & 0,500 & 45,376 & 0,750 \\
\hline
\end{tabular}


Tabela 2 - Análise Granulométrica da areia da praia de Bom Jesus dos Pobres - Bahia/Brasil, distribuída de acordo com o número da peneira e a abertura da malha, sendo demonstrados a massa retida e o diâmetro médio das partículas

(conclusão)

\begin{tabular}{llll}
\hline $\begin{array}{l}\text { Número da } \\
\text { peneira }\end{array}$ & $\begin{array}{l}\text { Abertura } \\
(\mathrm{mm})\end{array}$ & $\begin{array}{l}\text { Massa retida } \\
(\mathrm{g})\end{array}$ & $\begin{array}{l}\text { Diâmetro } \\
\text { médio }(\mathrm{mm})\end{array}$ \\
\hline 65 & $\mathbf{0 , 2 1 2}$ & $\mathbf{1 3 0 , 3 0 3}$ & $\mathbf{0 , 3 5 6}$ \\
80 & 0,180 & 11,046 & 0,196 \\
150 & 0,106 & 10,049 & 0,143 \\
270 & 0,270 & 0,416 & 0,188 \\
PAN & 0,00 & 0,00 & 0,135 \\
\hline Total & & 197,503 \\
\hline
\end{tabular}

Fonte: Dados da pesquisa.

A distribuição da areia, de acordo com a malha da peneira (Gráfico 5), evidencia o grau de seleção das partículas nas praias de Cabuçu e de Bom Jesus dos Pobres, demonstrando que os grãos estão na faixa de $0,750 \mathrm{~mm}$ a $0,356 \mathrm{~mm}$ de diâmetro.

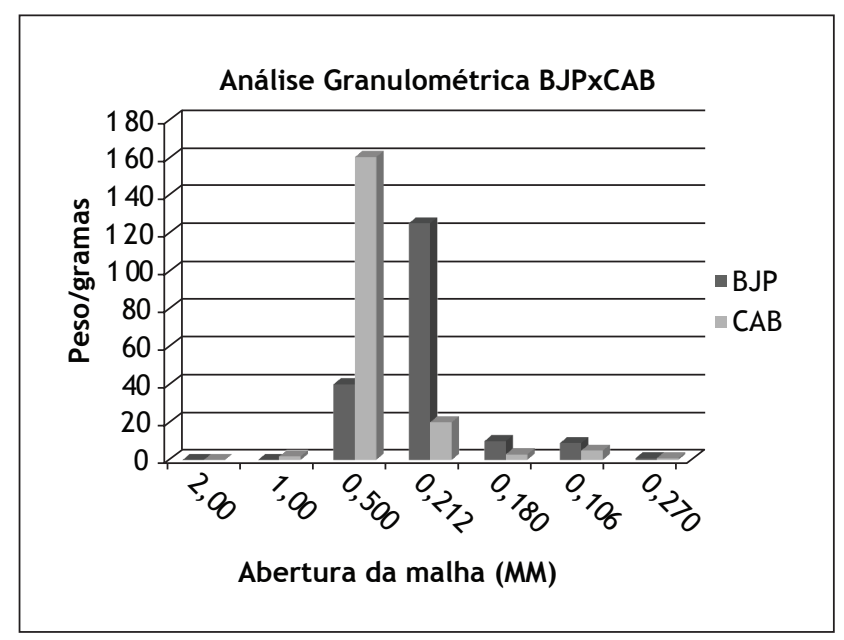

Gráfico 5 - Avaliação gráfica da distribuição do sedimento de acordo com a malha da peneira nas praias de Cabuçu e Bom Jesus dos Pobres - Bahia/Brasil

Fonte: Dados da pesquisa.

Segundo Pollock (14), em praias arenosas, as medianas dos grãos de 200 micrômetros ou menores, definem uma fauna intersticial verdadeira, admitindo-se que essa fauna pode habitar grãos bem selecionados com medianas entre 125 a 170 micrômetros. Bezerra et al. (13) já enfatizavam a influência do espaço intersticial disponível e sua oxigenação como fatores preponderantes na instalação e microdistribuição dos organismos meio faunísticos. Mostram que a influência de partículas finas pode limitar o desenvolvimento das populações, e ainda a forma do próprio grão de areia, admite a redução dos interstícios. Obviamente, granulometrias diferentes levam a uma qualidade de comunidades também diferenciadas.

\section{Conclusão}

As praias de Cabuçu e Bom Jesus dos Pobres apresentaram as variantes Físico-Químicas normais quando comparado com a Resolução Conama e outros autores. As variações paramétricas são associadas às condições climáticas da região do tipo tropical quente ao aporte de rios e energia da praia. Para as medidas de granulometria avaliadas nas praias de Cabuçu e Bom Jesus dos Pobres, a fração predominante nas peneiras foi areia média. Os sedimentos apresentaram pouca variação de diâmentro, caracterizando um maior grau de seleção das partículas. 0 espaço intersticial disponível e sua oxigenação, são fatores preponderantes na instalação e distribuição dos organismos no ambiente.

\section{Agradecimentos}

Aos professores: Dr. Thomas Vincent Gloaguen do Núcleo de Engenharia de Água e Solo (NEAS), e ao Dr. Sergio Schwarz da Rocha do Centro de Ciências Agrárias, Ambientais e Biológicas (CCAAB). Aos meus colegas e amigos: Alisson Sousa Matos Lima, Eduardo N. Souza Santos, Índira Oliveira da Luz, Adriana Freitas Pereira, Carlos A. de Oliveira e a Iranildes Paim Silva. Vocês foram de vital importância para conclusão deste trabalho.

\section{Referências}

1. Brown AC, Mclachlan A. Ecology of sandy shores. Amsterdam: Elsevier; 1990.

2. Amaral ACZ, Amaral EHM, Leite FPP, Gianuca NM. Diagnóstico sobre praias arenosas [online]. 2010 [acesso em 20 jan. 2010]. Disponível em: http://www.anp.gov. br/brasil-rounds/...r8/.../praias\%arenosas.pdf. 
3. Schmiegelow JMM. 0 planeta azul: uma introdução às ciências marinhas. Rio de Janeiro: Interciência; 2004.

4. Santos GS, Gonzalez P, Aguiar TR Jr., Adorno EV, De Carvalho AM. Identificação das conchas de gastrópodos ocupadas por paguros (Decapodas, Anomura, Paguroidea). Candombá. 2006;2(1):66-1.

5. Pasolini A. Análise morfodinâmica da praia de Marataízes frente a um possível processo de engordamento artificial [dissertação]. Vitória: Universidade Federal do Espírito Santo-Centro de Ciências Humanas e Naturais; 2005.

6. Tominaga EM, Rugno NC, Flynn MN. Processos hidrodinâmicos e sedimentares avaliados na região de São Sebastião - SP. In: anais do I Envieronmental and health world congress, 2006, Santos. Proceedings Environmental and health world congress, Santos, Barsil. Santos: Environmental and Health World Congress; 2006. p. 671-5.

7. Rocha-Barreira CA, Hijo CAG, Fernandes DAO, de Silva HL, Vidal JMA, Queiroz LR, et al. Levantamento da macroinfauna bentônica de ambientes inconsolidados do Estado do Ceará (faixa entre-maré de praias arenosas). Programa: Zoneamento Ecológico e Econômico (ZEE) da Zona Costeira do Estado do Ceará; 2005.

8. Farias NR. Caracterização físico-química nas águas da praia do Francês e praia do Saco, Marechal Deodoro Alagoas [dissertação]. Maceió: Universidade Federal de Alagoas - Instituto de Ciências Biológicas e da Saúde; 2007.
9. Ciências do Mar. Propriedades da água do mar [online] 2005 [acesso em 20 jan. 2010]. Disponível em: http// webserver.mohid.com/UnivLusofona/biomar1/Aulas/bm1_mod3.pdf.

10. Brandini F. A salinização costeira [online] 2008 [acesso em 20 jan. 2010]. Disponível em: http://www.oeco. com.br/frederico-brandini/19110-oecod228680.

11. Kaminski SM, Bersano JGF, Amaral WJA. Efeitos da salinidade e dieta alimentar sobre os Copépodes pseudodiaptomus richardi e Notodiaptomus incompositus em estudos de laboratório. Braz J Aquat Sci Technol. 2009;13(1):36-25.

12. McClintock JB. Pesquisador identifica que emissão de gás carbônico está tornando a água dos oceanos mais ácida [online] 2010 [acesso em 20 jan. 2010]. Disponível em: http://www.correiobraziliense.com.br/app/noticia.

13. Bezerra TNC, Genevois B, Fonsêca-Genevois VG. Influência da granulometria na distribuição e adaptação da meiofauna na praia arenosa do istmo de Olinda-PE. Oecol Bras. 1997(3):107-16.

14. Pollock LW. Ecology of intertidal meiobenthos. Smithson Contrib Zool. 1971;76:141-148. 
